Relations industrielles

Industrial Relations

\title{
Ressources humaines pour le développement industriel, Bureau international du travail, Genève, 1967, 276 pages.
}

\section{Bernard Solasse}

Volume 24, numéro 1, 1969

URI : https://id.erudit.org/iderudit/028003ar

DOI : https://doi.org/10.7202/028003ar

Aller au sommaire du numéro

Éditeur(s)

Département des relations industrielles de l'Université Laval

ISSN

0034-379X (imprimé)

1703-8138 (numérique)

Découvrir la revue

Citer ce compte rendu

Solasse, B. (1969). Compte rendu de [Ressources humaines pour le développement industriel, Bureau international du travail, Genève, 1967, 276 pages.] Relations industrielles / Industrial Relations, 24(1), 222-223.

https://doi.org/10.7202/028003ar

Tous droits réservés @ C Département des relations industrielles de l'Université Laval, 1969
Ce document est protégé par la loi sur le droit d'auteur. L'utilisation des services d'Érudit (y compris la reproduction) est assujettie à sa politique d'utilisation que vous pouvez consulter en ligne.

https://apropos.erudit.org/fr/usagers/politique-dutilisation/ 
comme information indispensable sur le marché du travail.

Finalement, l'article se termine par une série de recommandations, notamment, la nécessité de se tenir au courant des développements du marché du travail au moins annuellement comme on le fait aux Etats-Unis dans le "Manpower Report of the President". De plus, il souligne que des statistiques additionnelles devraient être fournies sur l'emploi et les salaires par occupation.

Dans les deux derniers articles Ian Drummond et David Stager examinent les relations entre le système éducationnel et les marchés du travail.

Pour le professeur Drummond les investissements dans l'éducation n'ont de sens que si leurs bénéfices sociaux dépassent leurs coûts sociaux. C'est pourquoi il présente différentes façons d'évaluer le rapport coûts/bénéfices et termine en proposant que l'analyse de ce rapport par les politiciens soit effectuée en conjonction avec les prévisions de l'évolution de la main-d'oeuvre.

David Stager, pour sa part, discute des difficultés conceptuelles qui existent lorsqu'on veut mesurer la « production 》 des institutions éducationnelles post secondaires. Cependant, il ne s'occupe que de l'aspect «instruction》 laissant de côté la «production》 de la recherche.

En terminant, il faut mentionner que ce livre est l'une des publications les plus intéressantes qui traitent des problèmes de main-d'oeuvre canadiens actuels.

\section{Jean BOIVIN}

Ressources humaines pour le développement industriel, Bureau international du travail, Genève, 1967, 276 pages.

Cette publication du BIT rassemble des documents préparés par le BIT en vue d'un colloque international de la Nouvelle organisation des Nations Unies pour le développement industriel (ONUDI).

Ces documents se répartissent en deux séries. Les documents de la première série, intitulée «Le problème de la maind'oeuvre » traitent de la formation professionnelle et de l'utilisation de la maind'oeuvre en vue du développement industriel.
La seconde série de documents intitulée \& autres questions exigeant une politique générale 》 porte «sur la participation sociale au développement industriel met l'accent sur l'importance d'une collaboration entre les différents groupes sociaux, tout particulièrement les employeurs, les travailleurs et leurs organisations, et de leur participation active au processus d'industrialisation, puis passe en revue les fins auxquelles cette participation serait utile et les formes qu'elle peut revêtir ».

Une première étude intitulée «Besoins en main-d'oeuvre qualifiée $\gg$ se conclut sur une série de recommandations pertinentes mettant l'accent sur les méthodes à employer afin de prévoir avec le maximum de précision l'évolution des besoins en main-d'oeuvre considérée non seulement sous l'angle quantitatif, mais également sous l'angle qualitatif (niveau de qualification professionnelle).

Une seconde étude consacrée aux programmes d'éducation et de formation préconise l'intégration sur la base de leur complémentarité de l'enseignement général et de l'enseignement technique et professionnel et tend à dégager les grandes lignes d'une méthodologie qui permettrait de dégager les objectifs généraux en matière d'éducation et de formation puis de les détailler ensuite en fonction des divers secteurs d'enseignement.

Cette étude comporte également diverses suggestions concernant l'organisation et le financement des divers programmes. Elle se termine sur quelques propositions précises de l'action internationale dans ce domaine.

Les deux dernières études de la première partie sont consacrées respectivement à « l'utilisation effective de la main-d'oeuvre en vue de l'industrialisation 》 et à la «formation professionnelle dans les petites entreprises de transformation des pays en voie de développement ».

La seconde partie contient une intéressante étude intitulée \&la participation sociale au développement industriel $\gg$.

«La mise sur pied d'une organisation satisfaisante des rapports humains et sociaux dans le secteur industriel et en particulier au sein des entreprises industrielles » est considérée comme une condition favorable au développement. 
De même celui-ci sera largement favorisé par un effort consenti par les groupements sociaux (syndicats de travailleurs, organisations patronales, mouvement coopératif, etc.)

L'on notera en particulier que cette étude préconise la mise en place de mécanismes de consultation et de collaboration dans les entreprises industrielles d'une certaine importance.

L'ensemble de ces études est étayé par d'abondantes références bibliographiques.

\section{Bernard SOLASSE}

The Mobile Manager: A Study of the New Generation of Top Executives, par E.E. Jennings, Bureau of Industrial Relations, Graduate School of Business Administration, The University of Michigan, 1967, 135 pages.

Les études faites sur la mobilité occupationnelle des dirigeants d'entreprises sont demeurées plutôt descriptives et comparatives. Elles seraient beaucoup plus utiles si elles permettaient de faire des prédictions sur les chances qu'aurait un jeune dirigeant d'accéder aux plus hauts postes de direction. Dans ce volume, l'auteur, professeur en administration des entreprises au Michigan State University, tente de jeter les bases d'une discipline nouvelle qui faciliterait ce genre de prédiction. Cette discipline s'appelle «the mobilography 》. Son objet réside dans l'étude des modèles de carrières des dirigeants en vue de connaître les caractéristiques de ceux qui sont mobiles, ceux qui ne le sont pas ou ne le sont plus momentanément. Elle étudie aussi les diverses avenues qu'empruntent les dirigeants pour atteindre le sommet de la pyramide sociale de l'entreprise.

Comme toute discipline possède un répertoire de concepts et un essai de classification, l'auteur fait un effort pour bien définir les concepts nouveaux qu'il se propose d'utiliser. De plus, il réussit à préciser les stages de la mobilité occupationnelle et à élaborer une classification des dirigeants en prenant comme critère le taux de mobilité ascendante.

Dans un premier chapitre traitant du «mobile hierarch », l'auteur souligne que les principaux facteurs qui peuvent expliquer un taux élevé de mobilité et qui constituent déjà les premiers résultats de recherche en «mobilography 》. Les chapitres qui suivent étudient plus en profondeur quelques-uns des principaux facteurs. En appendice, nous retrouvons un lexique des termes utilisés de même qu'un langage programmé développé par le professeur Jennings permettant à un ordinateur de colliger et traiter l'information contenue dans chaque «curriculum vitae 》 des membres de la haute-direction d'une entreprise.

Cette discipline serait utile aux dirigeants qui doivent assurer le relevé dans les postes de haute-direction. Elle aiderait ainsi à détecter chez les jeunes dirigeants, oeuvrant à des niveaux inférieurs les qualités et la compétence qu'il faut pour occuper des postes de haute-direction.

\section{Laurent BELANGER}

Management and the Behavioral Sciences, by Maneck S. Wadia, Allyn \& Bacon Inc., Boston, 1968, 544 pages.

Essentiellement, le rôle de l'administrateur prend un double aspect: d'abord celui d'obtenir que le travail devant être fait le soit et puis que le travail devant être fait le soit par des personnes autres que lui-même. Selon que l'on accordera plus d'importance à l'un ou l'autre de ces aspects, on sera affublé du vocable d'opérationnel ou de fanatique du comportement: l'école opérationnelle privilégie le processus d'administration comme étant le plus apte à la réalisation des buts de l'organisation, l'école du comportement lui rétorque par une argumentation basée sur l'importance de l'infinité d'influences exercées sur l'individu par le contexte interne et externe à cette même organisation.

L'espoir d'unifier ces conceptions dans une définition médiane de la fonction d'administrateur (processus qui consiste à atteindre les résultats désirés en influençant le comportement humain dans un contexte approprié) est la raison d'être et la justification de toutes ces études entreprises, de ces théories ébauchées en psycho-sociologie de l'entreprise. Ce volume écrit pour servir de manuel de classe aux étudiants gradués et sous gradués essaie de faire le point des connaissances actuelles, tente d'indiquer l'orientation des recherches futures. Le pro- 